ORIGINAL ARTICLE

\title{
Effect of universal adhesive on shear bond strength of metal bracket to enamel and dental amalgam
}

\author{
SHIVA ALAVI ${ }^{1}$, FATEME GHARIBPOUR ${ }^{2}$ \\ ${ }_{1}^{1}$ Associate Professor, Department of orthodontics/Dental Materials Research Center / Dental Research Institute/School of Dentistry/ Isfahan \\ University of Medical Sciences/ Isfahan/ Iran. Email address: alavi@dnt.mui.ac.ir, Orcid ld: 0000-0001-7288-4413 \\ ${ }^{2}$ post graduate, Dental students, Research Committee, Department of Orthodontics, School of Dentistry, Isfahan University of Medical \\ Sciences, Isfahan, Iran. Email address: fatemegharibpour@gmail.com, Orcid ID: 0000-0003-3913-7963 \\ Corresponding Author: Fateme Gharibpour, Department of Orthodontics, School of Dentistry, Isfahan University of Medical Sciences, \\ Isfahan, Iran. Phone numbers:+989365272382, E-mail address: fatemegharibpour@gmail.com
}

\begin{abstract}
background: the bond strength of bracket to the tooth substrate is a critical matter. The different surface treatments are introduced to achieve an appropriate bond strength. We aimed to investigate the shear bond strength(SBS) of metal brackets to amalgam surfaces with universal adhesive and compared the results with the enamel group.

Material and methods: in this in-vitro study, eighty specimens of amalgam in four groups and sixty specimens of the enamel in three groups were prepared. In amalgam groups, the control group was sandblasted and treated with transbond Xt whilst the other three groups prepared in the following order, group 2 sandblasted and treated with All Bond Universal(ABU), group 3 using diamond bur and $A B U$, and group 4 using self-etch mode of $A B U$. In enamel groups, control group was acid-etched and using transbond XT as a primer; in other two groups, the ABU was used as a primer in etched and self-etched mode. The specimens thermocycled and prepared for the SBS test. SEM analysis and ARI index were performed. pvalue $\leq$ was considered significantly.

Results: SBS of enamel was significantly higher than amalgam. Within enamel groups, etched with ABU showed the highest SBS. In amalgam group, the highest SBS was related to surface treated with ABU only, and the lowest SBS was related to diamond bur group.

Conclusion: The etched surface of enamel showed the appropriate bond strength to bracket, and in the amalgam group, using mechanical preparation can decrease the oxide layer and compromise amalgam SBS.

Keywords: Dental amalgam, Enamel, shear strength, Adhesive
\end{abstract}

\section{INTRODUCTION}

Orthodontic treatment has been an attractive dental treatment for clinical and aesthetic intention among teenagers through a long time(1); however, recent requests for a better look has been considerably increased (2); hence, orthodontic treatment has heightened among adults. Most adults have different prosthetic restorations such as amalgam in their mouths(3). In contemporary orthodontics, clinicians are used to band the brackets to different restorative materials(4).Since bonding can overcome the bands' obstacles and have been technically improved, demands for banding of posterior teeth has decreased. Achieving a reliable bond to restoration besides enamel is troublesome (4).

An ideal bonding of fixed appliances to any substrate should be resistant against the events in oral cavity.(5). Failure in bonding causes an interruption of treatment course, prolongs treatment time which is uncomfortable for patients and orthodontists (5).

Even though the range of 5-10 MPA was required for bonding to enamel surface(6), no counsel is currently available for other tooth substrates like amalgam; but from the clinical point of view, restorative materials should be at least as strong as enamel. (2)

In general, a multi-step adhesive system is most frequently used to attach brackets to enamel; although adequate bond strength would be obtained by this method, it takes a long time particularly in the first set up session(7). Also, this technique may make the teeth prone to tooth staining due to morphologically porous layers created by acid etching, as well as, white spot formation and decalcification (8) -the shallower etching pattern created by one-step adhesive, the lesser dental enamel desolvation(8)

The adhesive system's improvement intended to reduce technique sensitivity and decrease the chair times; in line with this tendency, the universal adhesive was introduced(9). This adhesive simplifies the bonding process and decreases the risk of damaging the enamel during debonding process(10).Acidic functional monomers such as MDP enable the bracket to endure applied forces and protect enamel integrity (11); furthermore, it is claimed that these materials can bond different tooth substrates such as enamel, dentin, amalgam, composite and porcelain(8).

The surface treatment is one of the most crucial parameters which affect the quality of treatment(12). Numerous mechanical and chemical acts have been established to enhance the bond strength of brackets to tooth; surface roughening like grinding by bur or sandblasting, surface conditioning similar to acid etching, using a chemical agent such as intermediate resin or metal bonding adhesive are some examples of surface treatment $(13,14)$

Hence, satisfactory bonding of bracket to amalgam is a clinician's issue(15). The current study objected to assessing the bond strength of metal bracket to amalgam with All Bond Universal (ABU) as a universal adhesive; moreover, the shear bond strength (SBS) of enamel as a control group were analyzed due to possess high bond strengths. 


\section{MATERIALS AND METHODS}

In this in-vitro study, bonding was performed on 140 surfaces, sixty human upper premolars, and eighty amalgam surfaces. Enamel (with three groups) as a control group and amalgam ( with four groups) as a testing group were examined. each groups contain 20 specimens.

Numerous test parameters can influence bonding values. All the parameters attempted to be standardized in the current study, except for the adhesive type to ensure a fair comparison. The teeth were extracted for the general dental reason concerning ethical guidelines; this represents residual biological material. The enamel surfaces were intact and free of any cracks or caries. All extracted teeth were kept in $0.5 \%$ tosylchloramide solution at room temperature due to yo DIN 13390-1 and DIN 1990-2 standards. The teeth were vertically mounted in a self-cure acrylic resin cylinder (figure 1) and cleaned using a rubber cap and slurry of non fluoridated pumice.

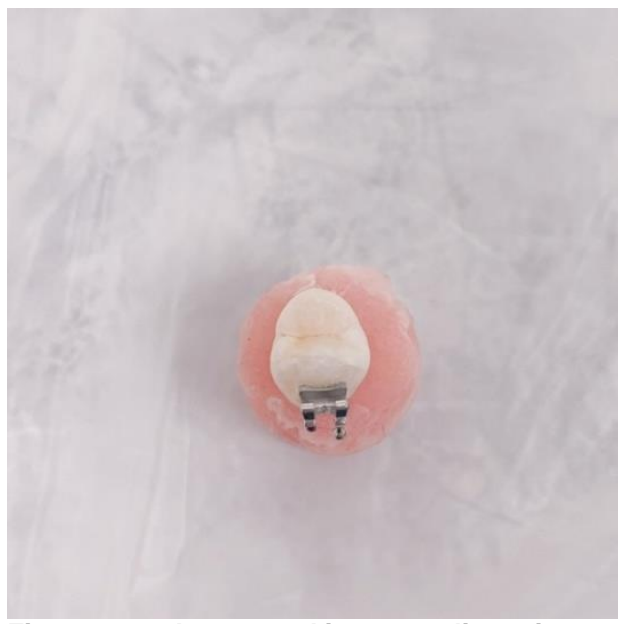

Figure 1 tooth mounted in an acrylic resin
The other eighty amalgam (ANA 2000, Angelholm, Sweden) specimens were prepared in clear plastic blocks ( figure 2) with a $6 \mathrm{~mm}$ width, $7 \mathrm{~mm}$ length, and $2 \mathrm{~mm}$ depth. Table 1 provide detail of the materials used.

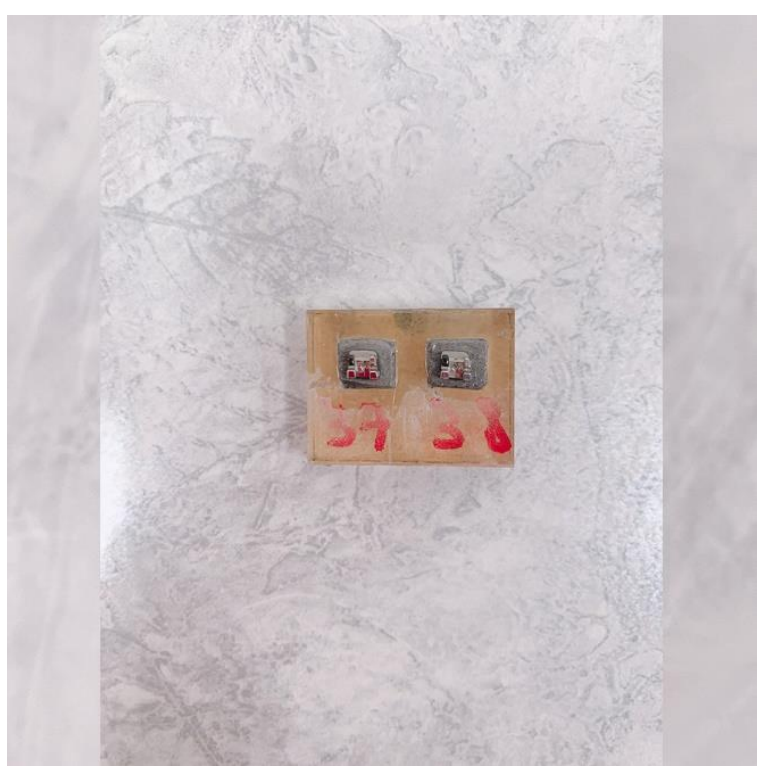

Figure 2 amalgam specimens prepared in clear plastic blocks

Table 1 materials used in this study and their compositions

\begin{tabular}{|l|l|l|}
\hline material & COMPOSITION & Lot number \\
\hline Transbond XT adhesive & TEGDMA, Bis-GMA & N861745 \\
\hline All Bond Universal adhesive & $\begin{array}{l}\text { MDP, Bis-GMA, HEMA, ethanol, water, } \\
\text { initiator }\end{array}$ & 1900004262 \\
\hline Transbond XT composite resin & SiO2 (99.61\%) & N864760 \\
\hline ANA 2000 amalgam & Non-gamma 2, lathe cut & $20-280$ \\
\hline
\end{tabular}

Sample preparation: there were three groups of enamel Group 1 ( etch + txt): as control, preliminary phosphoric etching $37 \%$ for $20 \mathrm{sec}$, water rinsing and drying, and application of transbond XT(TXT)( Transbond 3M, Unitek) for 2-3 sec and air drying gently.

Group 2 (etch + ABU): preliminary phosphoric etching 37\% for $20 \mathrm{sec}$, water rinsing and drying, and application of $\mathrm{ABU}$ (Bisco, USA) for 2-3 sec and air drying gently.

Group 3 ( Self-etch (SE) mode ABU): application of $A B U$, which was gently rubbed for 3-5 sec on the tooth surface and air drying subsequently.
After performing the corresponding surface treatment, in enamel group,stainless steel maxillary premolar brackets( Roth 018, Orth Organizer, USA) with a bracket base of 8.2 $\mathrm{mm}^{2}$ were bonded with a light-cured orthodontic adhesive composite resin( Transbond XT, 3M, Unitek) at the centre of clinical crowns. The adhesive resin was put onto the base of the bracket and then pressed onto the sample surface; a scaler was used to removing excess adhesive. All the samples were light-cured ( Woodpecker, LED, China) for $20 \mathrm{sec}(10 \mathrm{sec}$ mesial and $10 \mathrm{sec}$ distal) at a distance of 1-2 mm of the light tip from bracket margin by the portable light-curing device. 
In the amalgam group, they were divided into four groups. After preparing the amalgam in the cavities, the surface burnished with a hand instrument to obtain an almost smooth surface. The specimens were immersed in artificial saliva (Nikceram Razi, Isfahan, Iran) at $37^{\circ} \mathrm{C}$ for $24 \mathrm{~h}$ to induce oral cavity situation. After $24 \mathrm{~h}$, all the samples were prepared in the following order.

Group 1( sandblast + TXT): amalgam surfaces were sandblasted (Dento-prep, micro blaster, Ronvig, Denmark) with aluminium oxide $50 \mu \mathrm{m}$ abrasive powder for $3 \mathrm{sec}$ at a $10 \mathrm{~mm}$ distance with at approximately $7 \mathrm{~kg} / \mathrm{cm}$ of air pressure; then TXT as a primer for 2-3 sec applied and airdried gently for $1-2 \mathrm{sec}$.

Group 2( sandblast + ABU): amalgam surface was treated with aluminium oxide $50 \mu \mathrm{m}$ abrasive powder for 3 $\mathrm{sec}$ at a $10 \mathrm{~mm}$ distance with at approximately $7 \mathrm{~kg} / \mathrm{cm}$ of air pressure; then $\mathrm{ABU}$ as a primer for 2-3 sec performed and air-dried gently for $1-2 \mathrm{sec}$.

Group 3 ( bur+ABU): the surface was roughened with medium grit diamond bur( $D+Z$, Berlin, Germany) with highspeed handpieces with air and water for $5 \mathrm{sec}$ then airdried for 1-2 sec. We attempted to rough the surface with the same amount of pressure; then $A B U$ as a primer for 2-3 sec performed and air-dried gently for $1-2 \mathrm{sec}$

Group 4( $S E A B U)$ : samples were treated only by rubbing of $A B U$ for 3-5 sec and air-dried for 1-2 sec.

After performing the corresponding surface treatment, stainless steel central incisor bracket (Roth 018, Ortho Organizer, USA) with a bracket base of $9.5 \mathrm{~mm}^{2}$. With the same method of enamel group, all the test pieces were prepared by one person on one day.

All the samples ( enamel ad amalgam) were stored in water at $37{ }^{\circ} \mathrm{C}$ for $24 \mathrm{~h}$ in the incubator. after that, specimens were thermocycled (Delta TPO 2, Nemo, Mashhad, Iran) 1000 times from 5 to $55^{\circ} \mathrm{C}, 15 \mathrm{sec}$ in each bath, and 10 seconds travelling between two baths at room temperature. After thermocycling, debonding was performed with shearing forces using a testing machine( $\mathrm{K}$ 21046, Walter+bai, Switzerland). The shearing forcevelocity was $1 \mathrm{~mm} / \mathrm{min}$ with a load cell of 2.5 -kilogram newton(figure 3).

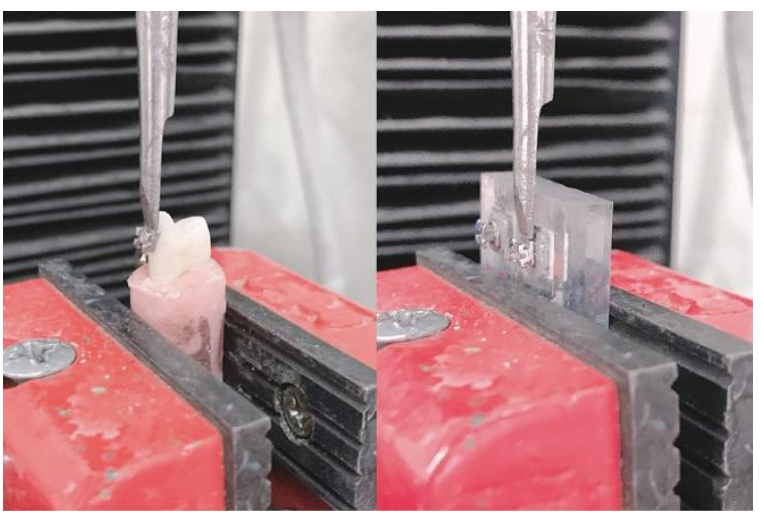

Figure 3 shear bond strength testing

The resulting force was converted into MPA in according via the following formula:

$$
R\left(N / \mathrm{mm}^{2}\right)=F(N) / A\left(\mathrm{~mm}^{2}\right),
$$

$\mathrm{R}=$ cohesive bond strength $/ \mathrm{F}=$ force $/ \mathrm{A}$ : cross-sectional surface of adhesive test

The remaining adhesive left on the base of the amalgam/enamel and bracket surface was assessed with microscopic magnification 10X ( SMP-200, HP, USA)to evaluate the ARI.as follows:

$0=$ no adhesive remaining;

$1=$ less than $50 \%$ of the adhesive remaining;

$2=$ more than $50 \%$ of the adhesive remaining;

$3=$ the whole adhesive remaining, showing bracket base impression(8).

One sample of each group was prepared for a Scanning electron microscope (INCAx-sight, England). The teeth were dehydrated and dissected, then sputter-coated with gold and observed under the microscope with magnification 2000; the amalgam sample has been mounted on aluminium moulds and observed under magnification 1000. Statistical analysis was carried out using IBM statistic 22(statistical software, SPSS for windows). Normal distribution was tested using Kolmogorov Smirnov, which showed that the value was not normally distributed. Nonparametric tests were therefore used; the statistical difference was analyzed using the Kruskal-Wallis test and Mann-Whitney adjusted with the Bonferroni test.

\section{RESULTS}

Descriptive statistics of SBS values expressed in MPa for the two adhesives and seven groups are presented in Table 2

Table 2 SBS test results of metal brackets to amalgam and enamel

\begin{tabular}{|c|c|c|c|c|c|c|}
\hline \multicolumn{7}{|l|}{ SBS MPA } \\
\hline & \multirow[t]{2}{*}{$\mathrm{N}$} & \multirow[t]{2}{*}{ Mean } & \multirow[t]{2}{*}{ Std. Deviation } & \multirow[t]{2}{*}{ Std. Error } & \multicolumn{2}{|c|}{ 95\% Confidence Interval for Mean } \\
\hline & & & & & Lower Bound & Upper Bound \\
\hline Amalgam- sandblast + TXT & 20 & 4.35 & 1.51 & .33 & 3.64 & 5.06 \\
\hline $\begin{array}{l}\text { Amalgam } \\
\text { Sandblast }+\mathrm{ABU}\end{array}$ & 20 & 4.48 & 1.06 & .23 & 3.98 & 4.98 \\
\hline Amalgam- bur+ $A B U$ & 20 & 2.95 & .99 & .22 & 2.48 & 3.42 \\
\hline $\begin{array}{l}\text { Amalgam } \\
\text { SE ABU }\end{array}$ & 20 & 6.19 & 1.53 & .34 & 5.47 & 6.91 \\
\hline $\begin{array}{l}\text { Tooth } \\
\text { Etch + TXT }\end{array}$ & 20 & 24.73 & 2.75 & .61 & 23.44 & 26.02 \\
\hline $\begin{array}{l}\text { Tooth } \\
\text { Etch +ABU }\end{array}$ & 20 & 31.97 & 2.83 & .63 & 30.64 & 33.29 \\
\hline $\begin{array}{l}\text { Tooth } \\
\text { SE ABU }\end{array}$ & 20 & 7.46 & 1.09 & .24 & 6.95 & 7.97 \\
\hline
\end{tabular}


Table 3 SBS of metal brackets to enamel analyzed with Mann-Whitney test

\begin{tabular}{|l|l|l|l|}
\hline & Group 1 & Group 2 & Group 3 \\
\hline Group 1(Etch + TXT) & - & $0.02^{*}$ & $0.01^{*}$ \\
\hline Group 2(Etch +ABU) & - & - & $0.001^{*}$ \\
\hline Group 3(SE ABU) & - & - & - \\
\hline
\end{tabular}

Groups annotated with * demonstrate significantly different result ( Mann-Whitney adjusted with Bonferroni)

Table 4 SBS of metal brackets to amalgam analyzed with Mann-Whitney test

\begin{tabular}{|l|l|l|l|l|}
\hline & Group 1 & Group 2 & Group 3 & Group 4 \\
\hline Group 1(sandblast + TXT) & - & 1 & $0.019^{*}$ & $0.05^{*}$ \\
\hline Group 2(Sandblast +ABU) & - & - & $0.01^{*}$ & $0.011^{*}$ \\
\hline Group 3(bur+ ABU) & - & - & - & $0.001^{*}$ \\
\hline Group 4(SE ABU) & - & - & - & - \\
\hline
\end{tabular}

Groups annotated with * demonstrate significantly different result ( Mann-Whitney adjusted with Bonferroni)

Table 5 adhesive remnant index (ARI) value of the adhesive system

Table 5 adhesive remnant index (ARI) value of the adhesive system
\begin{tabular}{|l|l|l|l|l|l|}
\hline group & ARI 0 & ARI 1 & ARI 2 & ARI 3 & ARI 4 \\
\hline E- (Etch + TXT) & - & 11 & 9 & - & - \\
\hline E-(Etch +ABU) & - & 13 & 7 & - & - \\
\hline E- (SE ABU) & 17 & 3 & - & - & - \\
\hline A- (sandblast + TXT) & 20 & - & - & - & - \\
\hline A- (Sandblast +ABU) & 20 & - & - & - & - \\
\hline A- (bur+ ABU) & 20 & - & - & - & - \\
\hline A- (SE ABU) & 20 & - & - & - & - \\
\hline
\end{tabular}

E: Enamel , A: amalgam

Table 6 ARI of metal brackets to enamel analyzed with Mann-Whitney test

\begin{tabular}{|l|l|l|l|}
\hline & Group 1 & Group 2 & Group 3 \\
\hline Group 1(Etch + TXT) & - & 1 & $0.001^{*}$ \\
\hline Group 2(Etch +ABU) & - & - & $0.001^{*}$ \\
\hline Group 3(SE ABU) & - & - & - \\
\hline
\end{tabular}

1Groups annotated with * demonstrate significantly different result (Mann-Whitney adjusted with Bonferroni)

The Kruskal-Wallis test exhibited that there were noteworthy differences within four amalgam groups and three enamel groups. $(p<0.001)$

Among the enamel group, all the subgroups showed considerable differences(shown in figure 4 and table 3 ), and the highest and the lowest SBS were related to group 2 and 3 , respectively.

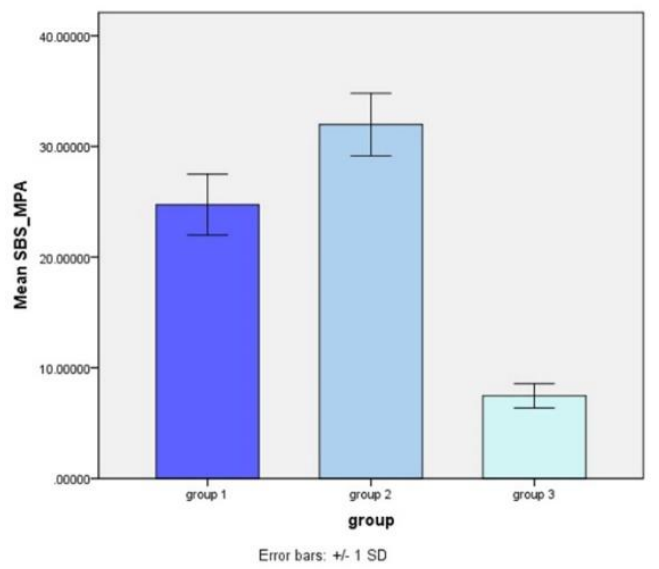

Figure 4 shear bond test results of enamel group
In amalgam groups, there were significant differences among surface treatments(shown in figure 5 and table 4).

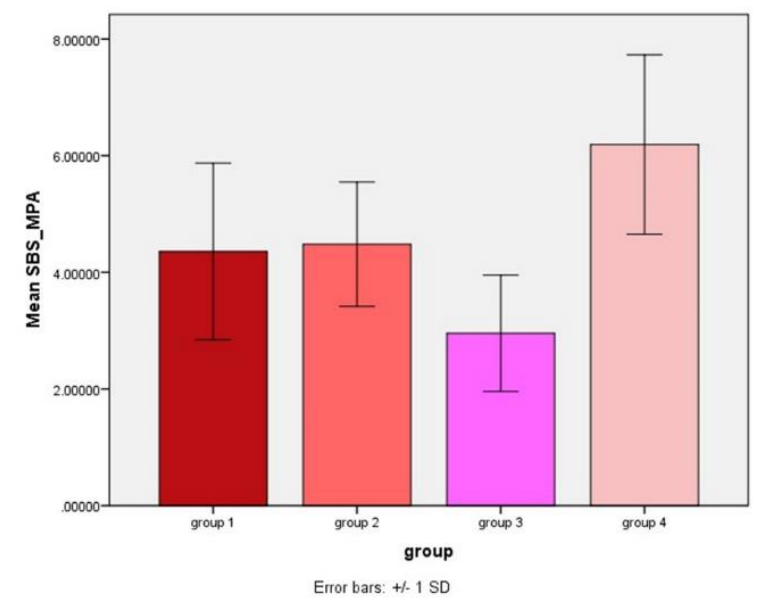

Figure 5 shear bond test results of amalgam group

Group 4 showed the highest SBS followed by two sandblasted groups, and the lowset SBS attributed to the 
group 3, also within sandblasted groups, group 2 showed higher SBS than group 1, but it was not significant.( $p: 1)$.

Enamel group showed statistically significant SBS value compare to amalgam using Mann-Whitney test, comparison of the group (SE ABU) in both enamel and amalgam showed significant difference which means enamel showed higher SBS than amalgam $(P<0.001)$.

The location of the fracture for each test sample was evaluated with the ARI index shown in table 5. In the enamel group and amalgam group, three and one types of bonding failures were identified, respectively.

In amalgam groups, all of the samples showed ARI 0 with no remnant adhesive on the amalgam surface. However, in the enamel group, the higher bond strength related to a higher index. Using the Man-Whitney test showed significant differences showed in table 6., comparison between group 1 and 2 was insignificant, but compared to group 3, ARI showed significant differences.

The SEM analysis of sandblasted amalgam (figure 6, 7)surfaces showed scratch lines which were rougher than two other types of treatment, and when primers were applied on both sandblasted and bur groups(figure 8), the surface appearance did not change considerably. In Selfetch mode of amalgam group(figure 9), a film thickness of resin could be observed. The SEM view of etched enamel groups(figure 10,11 ) showed micro retentive pattern and the resin tags, which penetrated to prismatic enamel, but SE specimens(figure 12) showed a honeycomb pattern and exposed enamel rods that not covered with resin.

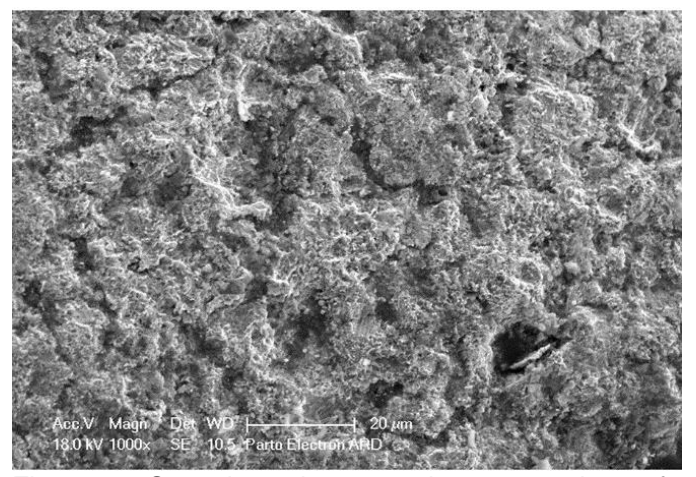

Figure 6 Scanning electron microscopy view of sandblasted amalgam surface Covered with transbond XT.(magnification: $1000 x)$ showing Scratch lines and irregularities.

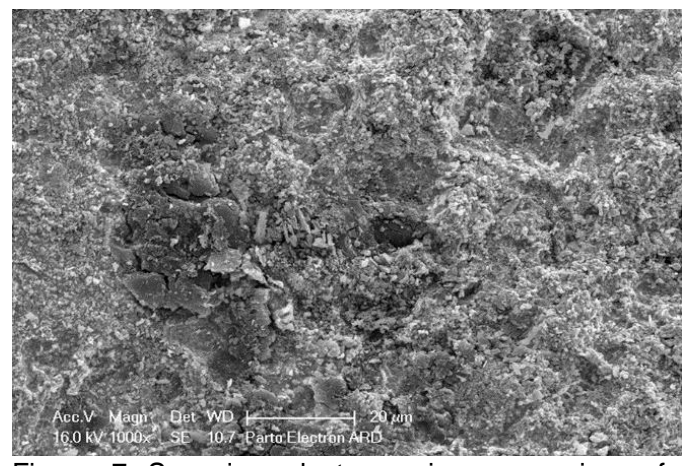

Figure 7 Scanning electron microscopy view of sandblasted amalgam surface Covered with universal adhesive.(magnification: 1000x) showing Scratch lines and irregularities, more resins can be observed

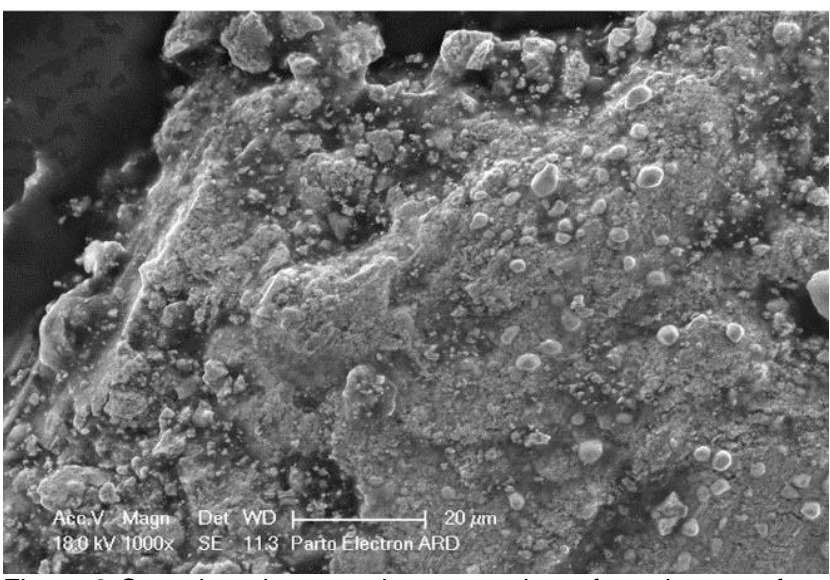

Figure 8 Scanning electron microscopy view of amalgam surface treated with diamond bur. (magnification: 1000x) less retentive patterns are observed

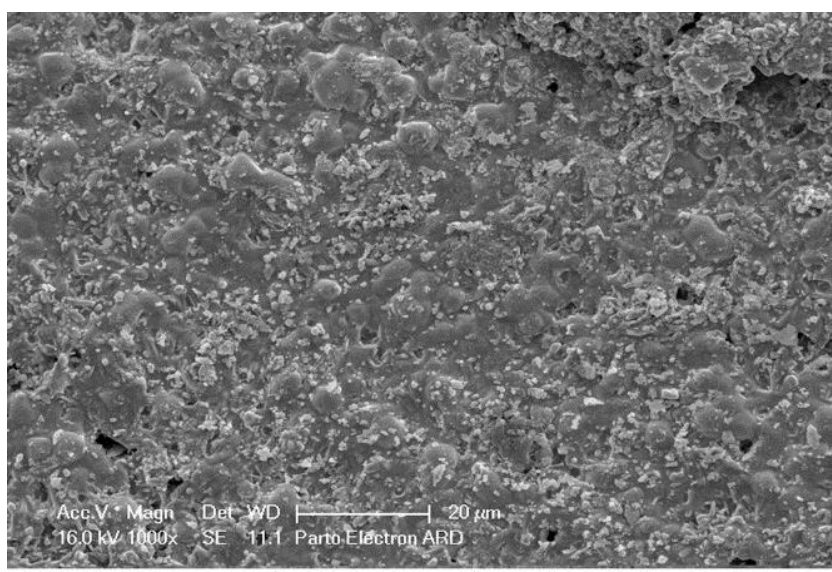

Figure 9 Scanning electron microscopy view of amalgam surface treated with universal adhesive.

(magnification : 1000x) showing a film thickness of resins that covered the amalgam surface

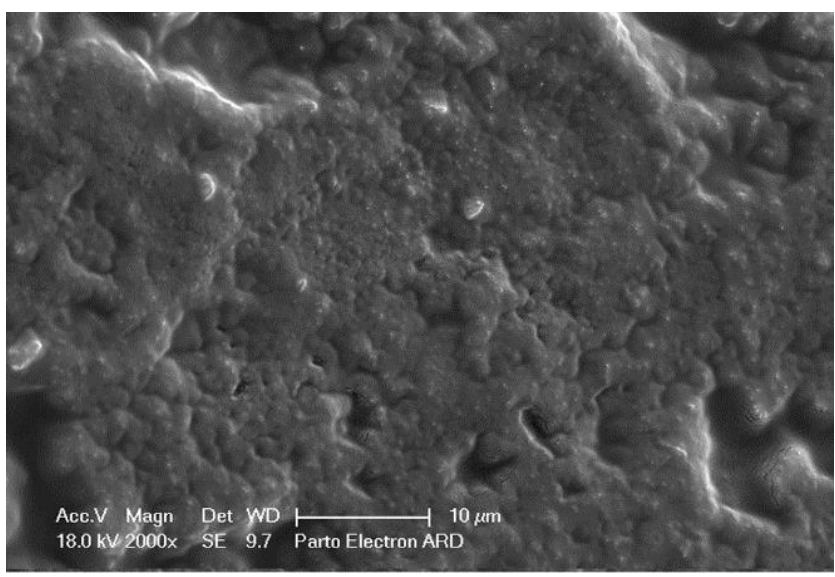

Figure 10 Scanning electron microscopy view of enamel surface etched with a transbond XT (magnification: 2000x) showing resin tags that penetrate to enamel rods and covered the surface 


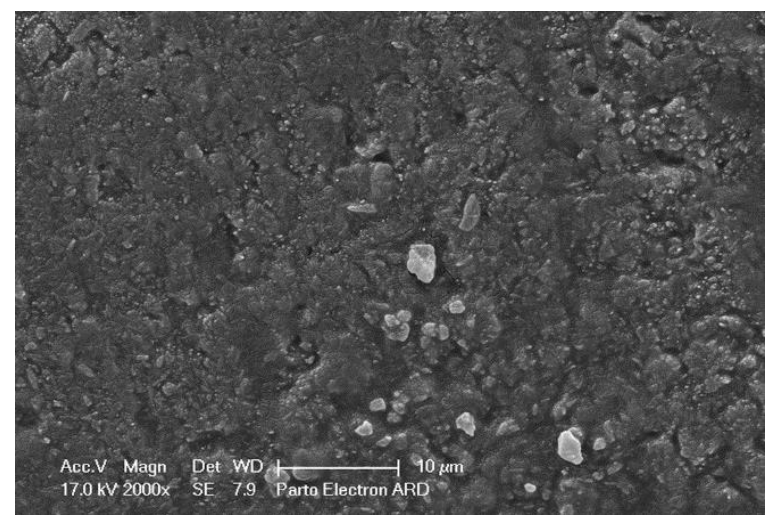

Figure 11 Scanning electron microscopy view of enamel surface etched with a universal adhesive (magnification: 2000x) showing resin tags that penetrate to enamel rods and covered the surface. More resins can be observed

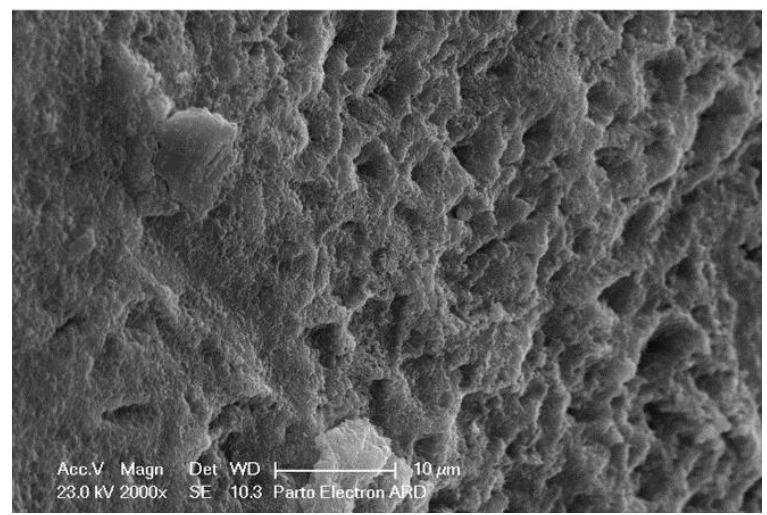

Figure 12 Scanning electron microscopy view of enamel surface self-etched with a universal adhesive (magnification: 2000x) showing honeycomb patterns and exposed enamel rods.

\section{DISCUSSION}

An ideal SBS of bracket to tooth substrate is to provide durable bonding throughout treatment without causing damage to enamel and restoration during debonding(5). Therefore, choosing an appropriate adhesive is noteworthy. $A B U$ was formulated to improve adhesion to normal and abnormal enamel surface, metal, and composite restoration(16). These materials contain acidic functional monomer giving them a high affinity to the calcium of hydroxyapatite to be applied to tooth either etched or without etched(8).

All three groups exhibited higher bond strength value in the enamel group than the minimum SBS required for clinical use. As Scribante et al. pointed out, an excellent orthodontic bonding should be able to tolerate masticatory forces (5-10 MPA), and the adhesive should not be too tough to destroy enamel integrity (40-50 MPa)(6). As the results showed, the higher SBS is related to group 2, followed by group 1 and group3. All values either fell in the mentioned range or were substantially higher. This result is consistent to the study conducted by Sena et al. (17). In both studies, the enamel group's SBS which followed by pre etching treatment was the highest, and the self- etches group significantly showed lower SBS, but clinically acceptable (17). This result is for the formation of long resin tags and micromechanical bond strength in etch groups(10).

The foremost step of enamel bonding was phosphoric acid etching for many years. The factor responsible for successful micromechanical bonding is the differential dissolution of enamel crystal and subsequent roughened surface (18). The smear layer will be removed by preliminary etching, causing enamel wettability, making hydroxyl groups of enamel exposed which lead to altering the surface properties from hydrophobic to hydrophilic. As hydrophilicity of enamel surface is crucial for compatibility between enamel and adhesive, chemical bonding will be improved by more hydrophilic adhesive like universal adhesive(9).

This study showed that SBS of universal adhesive was higher than conventional adhesive due to the study of Shafiei et al. (8). Even though TXT can be regarded as a standard adhesive system in orthodontic treatment which is an agent for assessing the bond strength of new products, it shows lower SBS compared to $\operatorname{ABU}(12,19)$. The presence of 10MDP as a functional monomer in the universal adhesive composition is the reason for its excellent performance(11).

Moreover, universal adhesive hydrophilicity is higher than the conventional one causing a better bond to enamel(9). Besides, the occurrence of a specific functional monomer (10 MDP) can ionically bond with the calcium ions of hydroxyapatite of uncut enamel which is hypermineralized, and form a non-soluble $\mathrm{ca}^{2+}$ salt which promotes strong adhesion(7). This chemical affinity is responsible for the low rate of dissolution and withstands ahead thermocycling(11).

Even though the most employed method is still primer acid conditioning, the application of self-etching despite restricted penetration into superficial enamel with shorter resin tags was recently improved (20). The formation of long resin tags may lead to some harm to hard tissue of enamel, inducing susceptibility to stain, white spot formation, increasing of decalcification, reduction in modulus of elasticity, and enamel hardness, and cracks formation(21).

Our study, as well as some other studies, proposes that SE group may have less, but adequate $\operatorname{SBS}(19,20$, $22,23)$. The SBS of SE group was $7.46 \mathrm{MPa}$ which is upper than the minimum range of SBS value obtained by Reynold et al. (24). Hellak et al. study, however, achieved a higher bond strength in SE group which may be explained by longer time of adhesive applying (20sec), besides they did not carry out the thermocycling process(25). As shown by Chu et al., more application time SE primer causes more SBS value(26).

Moreover, the enamel surface will lose its properties over time due to reaction with various ions and pores as well as occupying with foreign materials that create impurities in the enamel surface(2). This process is more prominent in extracted teeth, and it may be claimed that vital tooth in the oral cavity may show better SBS than extracted one, as can be seen in the study of Cal-Neto et al. (27).

Some dentists prefer to have higher bond strength since etched group's higher value supplies a safe margin against bracket debonding(8). 
On the other hand, SBS in all our amalgam groups were considerably weaker than the enamel group due to the absence of resin tags(12) which is consistent to finding other studies(1, 2, 15, 28, 29).

Understandably few tests have been performed on amalgam with universal adhesive. A comparison between the amalgam groups showed a noticeably significant difference in terms of different surface treatments.

In this in-vitro study, the universal adhesive which chemically bonded to the amalgam surface had higher SBS than other groups which seems clinically adequate. This higher value may be related to a superficial oxide layer on the amalgam surface and subsequent chemical reaction(29). However, MDP bond's mechanism is not entirely known, the involvement of chemical and mechanical retention is believed to be responsible(28).MDP monomer was initially designed to bond to metal oxide on pure base metal surface(30). This finding was also approved by Oskoee et al. study(15). In fact, mechanical treatment such as polishing or sandblasting seems to decrease the role of oxide layer(15). This finding is also mentioned in the study of Jost-Brinkham et al. (31).

Furthermore, ABU compromised of HEMA, MDP, and BisGMA. HEMA is a hydrophilic material which can decrease the surface tension, besides the increasing amount of HEMA result in increasing SBS, $40 \%$ of HEMA for nonprecious metal, and $30 \%$ for precious metal(1). Also, low viscosity of $A B U$ might be related to better wettability of amalgam, and consequently higher SBS value(32).

In this study, roughness and bond strength value seem to have no significant correlation; perhaps the main factor affecting amalgam bond strength is not surface area. The SBS value of sandblasted group of this study is similar to Zachrisson et al. (14) .Unlike finding the study of Germec et al., these differences may be related to resin application time on specimen's surface(29). Moreover, Wongsamut et al.'s study which examined other type of universal adhesive compared to sandblasting method indicated that SBS value was higher in sandblasted one(1). This difference can be related to the difference of adhesive or method of sample preparation or type of amalgam used (Kerr alloy). As Zachrisson et al. stated, bonding of composite resin to a spherical alloy is higher than admixed or lathe cut(14).

Along with other studies, the lowest SBS value caused when using a diamond bur as surface treatment(13, 14).

It should be mentioned that different factors affect SBS value, including the type of adhesive used, the bracket material properties, keeping method of test pieces, the adhesive gap diameter, test machines's shearing velocity, and type of dental restoration material(25). We tried to decrease these variables except adhesive type, any differences can be related to various SBS values achieved.

Due to the presence of enough sound enamel surrounding the buccal pit; therefore, a universal adhesive seems to provide adequate strength to resist the forces arising in orofacial region(14). Also, old amalgam restorations may pose an ample oxide layer, so the bond strength might be higher in the oral cavity(29).
This in-vitro study has some limitations including the absence of environmental factors such as $\mathrm{PH}$ changes, tooth brushing, bad oral habits. Also, the nature of forces in the oral cavity is complex and combines shear, tension, and compression. Alongside these limitations, the data from laboratory findings provide a guideline for a clinical condition(5). Finally, for future studies, an in-vivo study that is more reliable, using a different type of amalgam such as spherical or admixed and using different SE primer application time would be exiting.

\section{CONCLUSION}

Within the shortcoming of this in-vitro study, the following conclusion was extracted:

1) Although the enamel group's acid etching process showed the highest SBS in the conventional or universal group, the SE group also showed a promise SBS value.

2) Bond strength to amalgam surface depends on surface treatment technique

3) Universal adhesive and resin selection had a pronounced effect on amalgam bond strength; amalgam group's SE method provided a clinically acceptable bond force due to preserving oxide layer.

Therefore, universal adhesive containing 10-MDP gives sufficient bond strength to tooth substrate without requiring specific surface treatment such as sandblasting.

Acknowledgement

The authors thank the vice-chancellery of Isfahan University of Medical Sciences, Isfahan, Iran. For supporting research (grant \#399655). The manuscript relevant thesis of Fateme Gharibpour

Funding: Isfahan university of medical science. Grant \#399655

Conflict of interest: There is no conflict of interest.

\section{REFERENCES}

(1) Wongsamut W, Satrawaha S, Wayakanon K. Surface modification for bonding between amalgam and orthodontic brackets. J Orthod Sci. 2017;6(4):129-135.

(2) Alzainal AH, Majud AS, Al-Ani AM, Mageet AO. Orthodontic Bonding: Review of the Literature. Int J Dent. 2020:8874909.

(3) Stella JP, Oliveira AB, Nojima LI, Marquezan M. Four chemical methods of porcelain conditioning and their influence over bond strength and surface integrity. Dental Press J Orthod. 2015;20(4):51-56.

(4) Tahmasbi S, Badiee M, Modarresi M. Shear Bond Strength of Orthodontic Brackets to Composite Restorations Using Universal Adhesive. J Dent (Shiraz). 2019;20(2):75-82.

(5) Salama F, Alrejaye H, Aldosari M, Almosa N. Shear bond strength of new and rebonded orthodontic brackets to the enamel surfaces. J Orthod Sci. 2018;7:12.

(6) Scribante A, Contreras-Bulnes R, Montasser MA, Vallittu PK Orthodontics: Bracket Materials, Adhesives Systems, and Their Bond Strength. Biomed Res Int. 2016:1329814.

(7) Cerone M, El-Badrawy W, Gong SG, Prakki A. Bond Strength of Universal Self-Etch 1-Step Adhesive Systems for Orthodontic Brackets. J Can Dent Assoc. 2019;85:j6.

(8) Shafiei F, Sardarian A, Fekrazad R, Farjood A. Comparison of shear bond strength of orthodontic brackets bonded with a universal adhesive using different etching methods. Dental Press J Orthod. 2019;24(4):33.e31-33.e38.

(9) Tsujimoto A, Barkmeier WW, Takamizawa T, Watanabe $\mathrm{H}$, Johnson WW, Latta MA, et al. Influence of duration of phosphoric acid pre-etching on bond durability of universal 
adhesives and surface free-energy characteristics of enamel. Eur J Oral Sci. 2016;124(4):377-386.

(10) Rosa WL, Piva E, Silva AF. Bond strength of universal adhesives: A systematic review and meta-analysis. J Dent. 2015;43(7):765-776.

(11) Proença MAM, da Silva KTL, Costa ESA, Carvalho EM, Bauer J, Carvalho CN. Shear Strength of Brackets Bonded with Universal Adhesive Containing 10-MDP after 20,000 Thermal Cycles. Int J Dent. 2020:4265601.

(12) Golshah A, Mohamadi N, Rahimi F, Pouyanfar H, Tabaii ES, Imani MM. Shear Bond Strength of Metal Brackets to Porcelain Using a Universal Adhesive. Med Arch. 2018;72(6):425-429.

(13) Skilton JW, Tyas MJ, Woods MG. Effects of surface treatment on orthodontic bonding to amalgam. Aust Orthod J. 2006;22(1):59-66.

(14) Zachrisson BU, Büyükyilmaz T, Zachrisson YO. Improving orthodontic bonding to silver amalgam. Angle Orthod. 1995;65(1):35-42.

(15) Oskoee PA, Kachoei M, Rikhtegaran S, Fathalizadeh F Navimipour EJ. Effect of surface treatment with sandblasting and Er,Cr:YSGG laser on bonding of stainless steel orthodontic brackets to silver amalgam. Med Oral Patol Oral Cir Bucal. 2012;17(2):e292-296.

(16) Khanehmasjedi M, Naseri MA, Khanehmasjedi S, Basir L. Comparative evaluation of shear bond strength of metallic brackets bonded with two different bonding agents under dry conditions and with saliva contamination. J Chin Med Assoc. 2017;80(2):103-108.

(17) Sena LMF, Barbosa HAM, Caldas S, Ozcan M, Souza R. Effect of different bonding protocols on degree of monomer conversion and bond strength between orthodontic brackets and enamel. Braz Oral Res. 2018;32:e58.

(18) Türköz C, Ulusoy C. Evaluation of different enamel conditioning techniques for orthodontic bonding. Korean $\mathrm{J}$ Orthod. 2012;42(1):32-38.

(19) Hellak A, Ebeling J, Schauseil M, Stein S, Roggendorf M, Korbmacher-Steiner $\mathrm{H}$. Shear Bond Strength of Three Orthodontic Bonding Systems on Enamel and Restorative Materials. Biomed Res Int. 2016:6307107.

(20) Scougall-Vilchis RJ, Zárate-Díaz C, Kusakabe S, Yamamoto $\mathrm{K}$. Bond strengths of different orthodontic adhesives after enamel conditioning with the same self-etching primer. Aust Orthod J. 2010;26(1):84-89.
(21) Heravi F, Rashed R, Raziee L. The effects of bracket removal on enamel. Aust Orthod J. 2008;24(2):110-115.

(22) Holzmeier M, Schaubmayr M, Dasch W, Hirschfelder U. A new generation of self-etching adhesives: comparison with traditional acid etch technique. J Orofac Orthop. 2008;69(2):78-93.

(23) Scougall-Vilchis RJ, Ohashi S, Yamamoto K. Effects of 6 self-etching primers on shear bond strength of orthodontic brackets. Am J Orthod Dentofacial Orthop. 2009;135(4):424.e421-427; discussion 424-425.

(24) Reynolds I. A review of direct orthodontic bonding. Br.J.Orthod. 1975;2(3):171-178.

(25) Hellak A, Rusdea P, Schauseil M, Stein S, KorbmacherSteiner HM. Enamel shear bond strength of two orthodontic self-etching bonding systems compared to Transbond ${ }^{\mathrm{TM}} \mathrm{XT}$. J Orofac Orthop. 2016;77(6):391-399.

(26) Chu $\mathrm{CH}$, Ou KL, Dong de R, Huang HM, Tsai HH, Wang WN. Orthodontic bonding with self-etching primer and selfadhesive systems. Eur J Orthod. 2011;33(3):276-281.

(27) Cal-Neto JP, Miguel JA, Zanella E. Effect of a self-etching primer on shear bond strength of adhesive precoated brackets in vivo. Angle Orthod. 2006;76(1):127-131.

(28) Sperber RL, Watson PA, Rossouw PE, Sectakof PA. Adhesion of bonded orthodontic attachments to dental amalgam: In vitro study. Am J Orthod Dentofacial Orthop. 1999;116(5):506-513.

(29) Germec D, Cakan U, Ozdemir FI, Arun T, Cakan M. Shear bond strength of brackets bonded to amalgam with different intermediate resins and adhesives. Eur $\mathrm{J}$ Orthod. 2009;31(2):207-212.

(30) Akay C, Çakırbay Tanış M, Şen M. Effects of Hot Chemical Etching and 10-Metacryloxydecyl Dihydrogen Phosphate (MDP) Monomer on the Bond Strength of Zirconia Ceramics to Resin-Based Cements. J Prosthodont. 2017;26(5):419423.

(31) Jost-Brinkmann PG, Drost C, Can S. In-vitro study of the adhesive strengths of brackets on metals, ceramic and composite. Part 1: Bonding to precious metals and amalgam. J Orofac Orthop. 1996;57(2):76-87.

(32) Kanzow P, Baxter S, Rizk M, Wassmann T, Wiegand A. Effectiveness of a universal adhesive for repair bonding to composite and amalgam. J Oral Sci. 2019;61(2):343-350. 